\title{
Effect of shigella enterotoxin 1 (ShET1) on rabbit intestine in vitro and in vivo
}

\author{
A Fasano, F R Noriega, F M Liao, W Wang, M M Levine
}

\begin{abstract}
Background-Shigella enterotoxin 1 is a novel enterotoxin elaborated by Shigella flexneri 2 a that causes fluid accumulation in rabbit ileal loops and a rise in short circuit current in Ussing chambers.

Aims-To gain insights into the mechanism of action of shigella enterotoxin 1 .

Methods-Supernatants from genetically engineered clones either overexpressing shigella enterotoxin 1 or producing deletion mutants of the toxin were tested in rabbit ileum both in vitro and in vivo.

Results-In rabbit ileum shigella enterotoxin 1 induced an irreversible rise in short circuit current that was not mediated by any of the recognised intracellular mediators of secretion. Deletion of $90 \%$ of the $A$ subunit of the holotoxin ablated its enterotoxicity. In the in vivo perfusion model, the toxin induced a time dependent decrease in water absorption, whereas no changes were detected in the segment perfused with supernatants obtained from the deletion mutant. Finally, partially purified toxin induced a dose dependent increment in short circuit current that reached its plateau at a toxin concentration of $4 \times 10^{-6} \mathrm{M}$.

Conclusions-Shigella enterotoxin 1 induces a time and dose dependent intestinal secretion in the rabbit animal model, suggesting that it may be responsible for the watery phase of Shigella flexneri 2a infection.
\end{abstract}

Division of Pediatric Gastroenterology and Nutrition A Fasano

Center for Vaccine Development, University of Maryland, School of Medicine, Baltimore, MD 21201, USA A Fasano F R Noriega F M Liao W Wang M M Levine

Correspondence to: Dr Alessio Fasano, Gastrointestinal Pathophysiology Unit, Center for Vaccine Development, University of Maryland School of Medicine, $685 \mathrm{~W}$ Baltimore Medicine, 685 W Balt Room 465, Baltimore, MD 21201, USA

Accepted for publication 20 December 1996
(Gut 1997; 40: 505-511)

Keywords: diarrhoea, shigellosis, Ussing chambers, ileum, secretion.

Shigella dysenteriae type I was first isolated in 1898 during an epidemic of severe dysentery in Japan. ${ }^{1}$ Over the subsequent century other shigella species were identified, including Shigella flexneri, Shigella boydii, and Shigella sonnei, and the mechanisms whereby they cause disease have been intensively investigated. A cardinal feature of shigella virulence is the capacity of the organism to invade mammalian epithelial cells, followed by cell death, spread to adjacent cells, and elicitation of an intense inflammatory response with infiltration by polymorphonuclear leucocytes. $^{2-6}$ The classic dysenteric stool is scanty and consists of blood and mucus. However, most ill patients develop an initial phase of watery diarrhoea, that in mild cases may represent the only clinical presentation of the disease, without evolving to dysentery. ${ }^{78}$

We have recently reported the elaboration by shigella of two novel iron-regulated enterotoxins, shigella enterotoxin 1 (ShET1) and 2 (ShET2), that alter electrolyte and water transport in rabbit small intestine both in vitro and in vivo. ${ }^{9} 10$ ShET1 is a chromosomally encoded, $55 \mathrm{kDa}$ complex protein ${ }^{9}$ that is universally elaborated by Shigella flexneri $2 \mathrm{a}$ strains but only rarely by other serotypes. ${ }^{11}$ Sequencing analysis of the genes encoding this toxin disclosed the presence of two contiguous open reading frames (orfs) of $534(\operatorname{set} 1 \mathrm{~A})$ and 186 (set $1 \mathrm{~B}$ ) bp respectively, governed by the same promoter and separated by only $3 \mathrm{bp}$. ShET2 is a $62 \cdot 8 \mathrm{kDa}$ single protein that our group originally described in enteroinvasive Escherichia coli (EIEC) and referred to as EIEC enterotoxin (EIET). ${ }^{12}$ The gene encoding this toxin is located on the $140 \mathrm{mDa}$ invasiveness plasmid of shigella and seems to be present in more then $80 \%$ of a wide array of shigella serotypes examined. ${ }^{10}$ Gene sequence analysis showed that both ShET1 and ShET2 are genetically unrelated to shiga toxin elaborated by Shigella dysenteriae. Furthermore, the three toxins also seem to be immunologically unrelated.

In this paper we further characterise the mechanism of action of ShET1, both in vitro and in vivo. Our data indicate that ShET1 induces a time and dose dependent intestinal secretion in the rabbit animal model, suggesting that the toxin may have a role in the pathogenesis of the watery phase of dysenteric infection.

\section{Methods}

\section{OVEREXPRESSION OF ShET}

The $1093 \mathrm{bp}$ chromosomal fragment containing the ShET1 genes (set1) was cloned in the multiple copy plasmid pBluscript SK as previously described. ${ }^{9}$ The plasmid so obtained, pset 1, was transformed in $\mathrm{DH} 5 \alpha$ (Fig 1) and overexpression of the ShET1 protein was obtained in iron depleted culture supernatants. ${ }^{9}$ More specifically, $100 \mathrm{ml}$ Luria Bertani broth containing $25 \mu \mathrm{g} / \mathrm{ml}$ of the iron chelator ethylenediamine-N-N' diacetic acid (EDDA) and $100 \mu \mathrm{g} / \mathrm{ml}$ ampicillin were inoculated with a single colony of $\mathrm{DH} 5 \alpha$ (pset 1 ) and incubated overnight at $37^{\circ} \mathrm{C}$ with shaking (200 rpm). Bacterial cells were removed by 

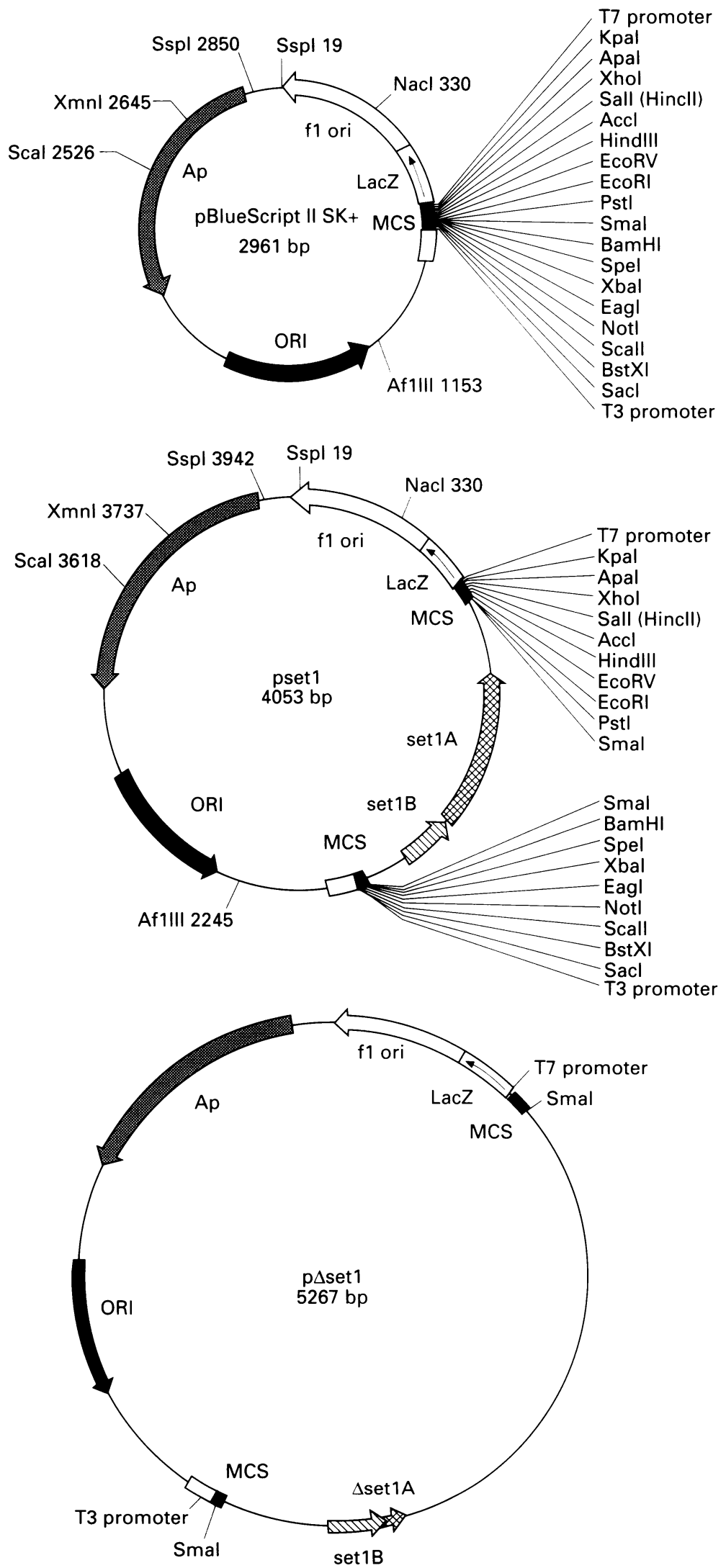

Figure 1: Genetic construction of DH5 $\alpha$ ( $p$ set 1$)$ and its deletion mutant DH5 $\alpha$ ( $p \Delta \operatorname{set} 1)$. Plasmid $p$ Bluscript was used to mobilise the $1098 \mathrm{bp}$ Shigella flexneri 2 a chromosomal fragment containing the set $1 A$ and set $1 B$ orfs. The deletion mutant was engineered by deleting $90 \%$ of the set $1 A$ gene (from $A A$ residue 17 to $A A$ redisue 169), while the natural promoter and the whole set $1 B$ orf were left unaltered.

centrifugation, and the supernatant was filtersterilised through a $0 \cdot 22 \mathrm{~mm}$ membrane filter. The supernatant was then frozen until used for the experiments described later.
PURIFICATION OF ShET1

Overexpressed ShET1 was obtained as described above. The proteins contained in the $\mathrm{DH} 5 \alpha$ (pset 1 ) supernatant were then precipitated with saturated trichloroacetic acid (TCA) $(20: 80 \mathrm{v} / \mathrm{v})$ and separated by SDS-PAGE electrophoresis. Resultant bands were transferred to a nitrocellulose membrane and cut into two strips to be either silver stained to visualise protein bands or reacted with rabbit antiserum against ShET $^{9}$ for western immunoblotting. The single band corresponding to the $55 \mathrm{kDa}$ ShET 1 protein was carefully cut out of the gel and electroeluted using an electroseparation chamber (Schleicher and Schuell, Keene, NH, USA). Purity of ShET1 was established by SDS-PAGE electrophoresis and by western immunoblotting. The amount of electroeluted ShET 1 was determined by the Bradford method. ${ }^{13}$

CONSTRUCTION OF A set 1 DELETION MUTANT A $\Delta$ set 1 allele was obtained by deleting $90 \%$ of set $1 \mathrm{~A}$ gene (from AA residue 17 to $\mathrm{AA}$ residue 169), while the natural promoter and the whole B subunit were left intact (Fig 1). DNA segments were amplified from a library derived phagemid $\mathrm{pF} 9-1-90^{9}$ containing the $\Delta$ set 1 allele and fused by polymerase chain reaction (PCR). Unique restriction sites were then introduced using external primers and were utilised to clone the $\Delta$ set 1 allele into pBluscript $\mathrm{SK}$. The plasmid so obtained (named $\mathrm{p} \Delta \operatorname{set} 1$ ) was electroporated in $\mathrm{DH} 5 \alpha$ and supernatants of this strain (DH5 $\alpha(\mathrm{p} \Delta$ set 1$))$ (Fig 1) were prepared as reported above and frozen until tested in the in vitro and in vivo assays described below. The amount of protein present in $\mathrm{DH} 5 \alpha$ ( $\mathrm{p} \Delta$ set 1 ) was determined by the method of Bradford ${ }^{13}$ and results were comparable with the total protein concentration present in the $\mathrm{DH} 5 \alpha$ (pset 1 ) supernatant $(4 \cdot 21 \mu \mathrm{g} / \mathrm{ml} v 4.41 \mu \mathrm{g} / \mathrm{ml}$ respectively).

CONSTRUCTION OF MALTOSE BINDING PROTEIN (MBP)-ShET1 (A SUBUNIT FUSION PROTEIN) AND PREPARATION OF ANTI-A SUBUNIT POLYCLONAL ANTIBODIES

The ShET1 A subunit gene was amplified by PCR using pset 1 as a template. Fidelity of PCR amplification was confirmed by DNA sequencing of the plasmid insert. set $1 \mathrm{~A}$ gene was then fused in frame with the maltose binding protein (MBP) gene, using vector pMalc $2^{14}$ to create a MBP-ShET1 A fusion protein. The fusion product was expressed in Escherichia coli and obtained by disrupting the cells using a French press. The cell lysate was centrifuged for 30 minutes at $15000 \mathrm{~g}$, and the supernatant was filtered, diluted five times in buffer A $(20 \mathrm{mM}$ Tris $\mathrm{HCl} \mathrm{pH} \mathrm{7.5,} 1 \mathrm{mM}$ EDTA, $0.2 \mathrm{M} \mathrm{NaCl}, 1 \mathrm{mM} \mathrm{NaN}_{3}$, and $1 \mathrm{mM}$ 2-ME), and loaded on to an amylose column (New England Biolabs) at a flow rate of $1 \mathrm{ml} /$ min. The column was washed with five volumes of buffer $A$ and the fusion protein was eluted with $10 \mathrm{mM}$ maltose in buffer $A$. Protein concentrations were assessed with a 
Bio-Rad protein assay, and purity was determined by SDS-PAGE followed by Comassie or silver staining.

To raise polyclonal antibodies against ShET1 A subunit, purified MBP-ShET1 A fusion protein was mixed with an equal volume of complete Freund's adjuvant and injected into an adult male New Zealand white rabbit. Two booster doses were given with Freund's incomplete adjuvant four and eight weeks later, and the animal was then bled.

USSING CHAMBERS

Experiments were carried out as previously described. ${ }^{15}$ Briefly, adult male New Zealand white rabbits (body weight $2-3 \mathrm{~kg}$ ) were killed by cervical dislocation. A $20 \mathrm{~cm}$ segment of ileum was removed, rinsed free of the intestinal content, opened along the mesenteric border, and stripped of muscular and serosal layers. Eight sheets of mucosa so prepared were then mounted in lucite Ussing chambers $\left(1.12 \mathrm{~cm}^{2}\right.$ opening), connected to a voltage clamp apparatus (EVC 4000 WPI, Saratosa, FL, USA), and bathed with freshly prepared buffer containing (in $\mathrm{mM}$ ): $\mathrm{NaCl}, 53 ; \mathrm{KCl}, 5 ; \mathrm{Na}_{2} \mathrm{SO}_{4}$, 30.5; mannitol, 30.5; $\mathrm{Na}_{2} \mathrm{HPO}_{4}, \quad 1.69$; $\mathrm{NaH}_{2} \mathrm{PO}_{4}, \quad 0 \cdot 3 ; \mathrm{CaCl}_{2} 1 \cdot 25 ; \mathrm{MgCl}_{2} 1 \cdot 1$ $\mathrm{NaHCO}_{3}, 25$. The bathing solution was maintained at $37^{\circ} \mathrm{C}$ with water jacketed reservoirs connected to a constant temperature circulating pump and gassed with $95 \% \mathrm{O}_{2} / 5 \% \mathrm{CO}_{2}$. Potential difference (PD) and short circuit current (Isc) were measured under short circuit conditions and tissue resistance (Rt) was calculated as previously described. ${ }^{16}$ Filtered supernatants $(300 \mu \mathrm{l})$ obtained from either $\mathrm{DH} 5 \alpha$ (pset 1$)$ or $\mathrm{DH} 5 \alpha$ ( $\mathrm{p} \Delta$ set 1$)$ were added to the mucosal surface and an identical amount was added to the serosal surface to preserve the osmotic balance. In selected experiments, partially purified ShET1 was added only to the mucosal side of the rabbit ileum. Variations in PD, Isc, and Rt were then recorded every 10 minutes. At the end of every experiment, 0.5 $\mathrm{mM}$ glucose was added to the mucosal side of each chamber. Only those tissues which showed an increase in Isc in response to glucose, indicating tissue viability $(98 \%$ of the tissues tested), were included in the analysis.

IN VIVO PERFUSION ASSAY

Intestinal perfusion was carried out according to the method previously described by Sladen et $a l^{17}$ with minor modifications. More specifically, after a 24 hour fast, $2 \cdot 5-3 \mathrm{~kg}$ adult male New Zealand white rabbits were anaesthetised with $50 \mathrm{mg}$ ketamine/kg body weight, followed by intramuscular injection of $7.0 \mathrm{mg}$ xylazine/ $\mathrm{kg}$ body weight. Their body temperature was kept at $37^{\circ} \mathrm{C}$ by a lamp. The abdominal cavity was opened by a midline incision and two distinct segments of jejunum were cannulated. A second cannula was placed $10-15 \mathrm{~cm}$ below each proximal cannula. The segments were rinsed free of intestinal contents with $0.9 \%$ (w/v) $\mathrm{NaCl}$ warmed to $37^{\circ} \mathrm{C}$. The proximal cannulae were connected by a polyvinyl tube to a peristaltic pump (model WPI SP220 I), and the two segments were perfused at a rate of $0.4 \mathrm{ml} / \mathrm{min}$ with a solution comprising 2.0 $\mathrm{mmol} / \mathrm{l}$ glucose, $4.0 \mathrm{mmol} / \mathrm{KCl}, 25 \mathrm{mmol} / \mathrm{l}$ $\mathrm{NaHCO}_{3}, 3.0 \mathrm{~g} / 1 \mathrm{PEG}-4000$, and $3.0 \mu \mathrm{Ci} / 100$ $\mathrm{ml}{ }^{14} \mathrm{C}-\mathrm{PEG}-4000$. The solution was made isotonic by adjustment with $\mathrm{NaCl}$, and the $\mathrm{pH}$ fixed at $7 \cdot 4$ by gassing with $95 \% \mathrm{O}_{2} / 5 \% \mathrm{CO}_{2}$.

Eluates were collected in 20 minute aliquots from the distal cannulae. An initial equilibrium period of 30 minutes was allowed, followed by three consecutive 20 minute collection periods for baseline measurement of the net transport of water and electrolytes in each segment studied.

Subsequently, one of the two segments was perfused with the same solution described above containing $30 \mu \mathrm{l} / \mathrm{ml} \mathrm{DH} 5 \alpha$ (pset 1) supernatant, while the second segment was perfused with a similar amount of $\mathrm{DH} 5 \alpha$ ( $\mathrm{p} \Delta$ set 1$)$ supernatant. A second perfusion period (30 minute equilibration plus $6 \times 20$ minute collection) was then carried out. At the end of the experiment, the animal was killed and the segments perfused were isolated, measured, dried, and weighed. Water absorption was then calculated as previously described. ${ }^{17}$

\section{Results}

INTRACELLULAR MESSENGER(S) OF ShET 1 ENTEROTOXIC ACTIVITY

To establish whether the enterotoxic effect of ShET1 was mediated by one of the described intracellular mediators of intestinal secretion (cAMP, cGMP, and $\mathrm{Ca}^{2+}$ ), $5 \mathrm{mM}$ theophylline, $0.2 \mathrm{mM} 8 \mathrm{Br}$-cGMP, and $5 \times 10^{-7} \mathrm{M} \mathrm{Ca}$ ionophore A23187 were tested in Ussing chambers in the presence and absence of DH5 $\alpha$ (pset 1) supernatant. Segments of rabbit ileum mounted in Ussing chambers were paired based on their Rt. Supernatant obtained from $\mathrm{DH} 5 \alpha$ (pset 1 ) (positive control) was added to one of the chambers, while the coupled chamber was exposed to DH5 $\alpha$ (pBS) (negative control) supernatant. Once the increment of Isc induced by $\mathrm{DH} 5 \alpha$ (pset 1 ) reached a plateau, the second messenger to be tested was added to the serosal side of both chambers and the subsequent increment in Isc recorded. As shown in Fig 2, the Isc changes induced by the three intracellular messengers tested were similar in the ShET1 exposed and control exposed tissues, suggesting that none of them is involved in ShET1 secretory action.

\section{LACK OF REVERSIBILITY OF ShET 1}

ENTEROTOXIC EFFECT

To establish whether the ShET1 enterotoxic effect was reversible, rabbit ileal mucosa was first exposed to $\mathrm{DH} 5 \alpha$ (pset 1 ) supernatant. When the enterotoxic effect of supernatant containing ShET1 reached its plateau, the reservoir was emptied, rinsed twice with Ringers' solution, and refilled with Ringers' alone. No substantial changes in Isc were seen after ShET1 withdrawal (Fig 3), suggesting either an irreversible binding of the toxin with its receptor 


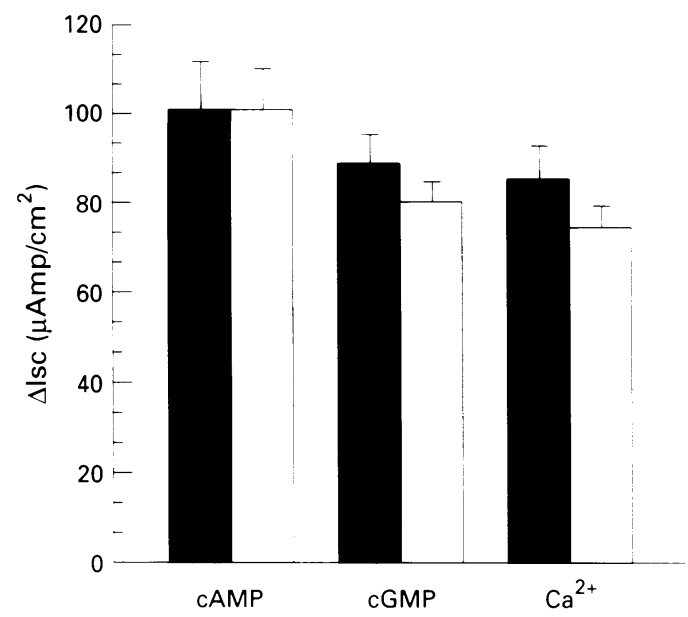

Figure 2: Peak increments in Isc after addition of 5 mmol/ theophylline (cAMP), $0.2 \mathrm{mmol}$ 8-bromo-cGMP (cGMP), or $5 \times 10^{\circ}$ moln calcium ionophore $A 23187$ $\left(\mathrm{C}^{2+}\right)$ to tissues pretreated with either DH5 $\alpha$ (pset 1)

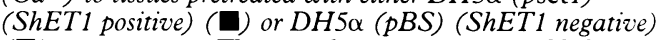
( $\square$ ) supernatants. The second messengers were added to the serosal side once the Isc increment induced by ShET reached the plateau (110-120 $\mathrm{min})(n=4$ animals). Bars represent SEM.

or a continuous activation of the intracellular signalling that mediates the enterotoxin effect.

WESTERN IMMLNOBLOTS OF DH $5 \alpha$ (pset 1$)$ AND ITS DELETION MUTANT DH $5 \alpha(\mathrm{p} \Delta$ set 1$)$

Western blotting performed with the rabbit antiserum raised against $\mathrm{ShET} 1^{9}$ showed the $55 \mathrm{kDa}$ ShET 1 band in DH5 $\alpha$ (pset1) supernatant, but not in $\mathrm{DH} 5 \alpha(\mathrm{p} \Delta$ set 1$)$ supernatant (Fig 4A). Interestingly, the band was present only in the supernatant but not in the cell lysate of $\mathrm{DH} 5 \alpha$ (pset 1), suggesting that ShET1 is a secreted protein. These results are in keeping with the fact that the predicted amino acid

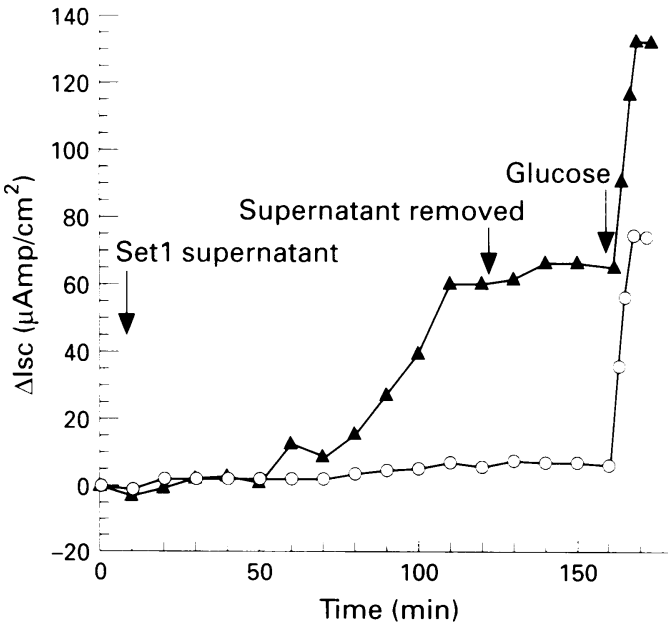

Figure 3: Lack of reversibility of ShET1 enterotoxic effect. Supernatants obtained from both $D H 5 \alpha$ (pset 1) (ShET

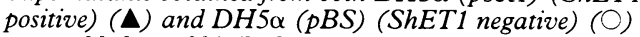
were added to rabbit ileal mucosa mounted in Ussing chambers and changes in Isc were followed over time. ShET1 induced a time dependent increase in Isc compared with the negative control that reached its plateau after almost 100 minutes. Once the toxin was withdrawn from the reservoir (arrow), no modifications in Isc were observed, suggesting an irreversible binding of ShET1 to its receptor. Addition of $0.5 \mathrm{mM}$ glucose to the mucosal side of the rabbit ileum induced a similar rise in Isc in both the ShETI and negative control exposed tissues, proving that ShET1 did not affect the tissue viability ( $n=4$ animals). sequence of the set 1 gene features a putative signal sequence. ${ }^{9}$ As these experiments were performed under denaturing conditions, we repeated the western blotting experiment using non-denaturing conditions. The results showed in Fig 4B confirmed that ShET1 is present in DH5 $\alpha$ (pset 1) supernatant, but is lacking in both $\mathrm{DH} 5 \alpha$ (pBS) and $\mathrm{DH} 5 \alpha$ ( $\mathrm{p} \Delta$ set 1$)$ supernatants. Finally, to establish whether DH5 $\alpha$ ( $\mathrm{p} \Delta$ set 1$)$ expresses the ShET1 $B$ subunit, the western blotting was repeated using polyclonal antibodies raised against the ShET1 A subunit and compared with western immunoblots performed using polyclonal antibodies raised against the entire holotoxin. ${ }^{9}$ As shown in Fig 4C, the polyclonal antibodies against the holotoxin visualised a $35 \mathrm{kDa}$ band present in both $\mathrm{DH} 5 \alpha$ (pset 1) and $\mathrm{DH} 5 \alpha$ ( $\mathrm{p} \Delta$ set 1 ) supernatants, but not in $\mathrm{DH} 5 \alpha$ (pBS) negative control supernatant. This band was not visualised by the polyclonal antibodies raised against the ShET1 A subunit, suggesting that the $35 \mathrm{kDa}$ band may represent a $\mathrm{B}_{5}$ aggregate and, therefore, that the $B$ subunit is still expressed by the deletion mutant.

EFFECT OF ShET 1 DELETION MUTANT IN USSING CHAMBERS

The apparent molecular size of ShET 1 by both gene sequencing and western blot analysis ${ }^{9}$ suggests a holotoxin stoichiometry $\left(\mathrm{A}_{1}-\mathrm{B}_{5}\right)$ similar to that of other well established enterotoxins. ${ }^{18-21}$ To establish whether the ShET $1 \mathrm{~A}$ subunit plays a pivotal role in ShET1 enterotoxicity, supernatants obtained from $\mathrm{DH} 5 \alpha$ ( $\mathrm{p} \Delta$ set 1 ) lacking $90 \%$ of ShET 1 A subunit were tested in Ussing chambers. When added to rabbit ileal mucosa, this supernatant failed to induce the increase in Isc typically seen with DH5 $\alpha$ (pset 1 ) supernatant (Fig 5).

IN VIVO PERFUSION EXPERIMENTS

We have previously shown that ShET1 containing supernatants induce a mild, but significant fluid accumulation in rabbit ileal loops in vivo. ${ }^{9}$ To better define the mechanism of secretion of ShET 1 and to establish the in vivo effect of the deletion mutant of ShET 1 protein, two contiguous segments of jejunum were simultaneously perfused in the same animal with a saline solution (period 1), followed by a second period during which solutions containing either $\mathrm{DH} 5 \alpha$ (pset 1 ) or $\mathrm{DH} 5 \alpha$ ( $\mathrm{p} \Delta$ set 1$)$ supernatants were perfused. The first period of perfusion was characterised by the active absorption of water and electrolytes in both segments (Fig 6). After 60 minutes the addition of ShET1 to the perfusion solution produced a significant decrease in water absorption, whereas no changes were detected in the segment perfused with ShET1 protein deleted of its A subunit (Fig 6).

DOSE-RESPONSE CURVE OF ShET 1

ENTEROTOXICITY

To establish whether the enterotoxic effect of ShET1 was dose dependent, increasing 


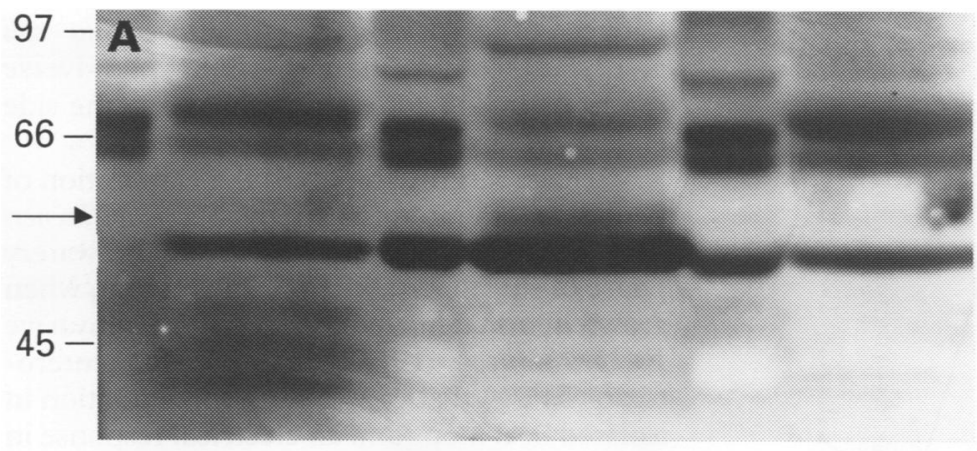

A B

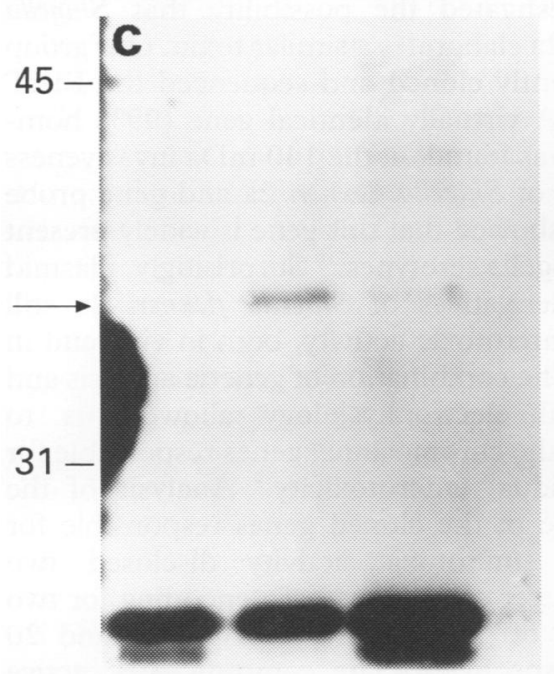

A

B

$\mathrm{C}$

D

$E$

F

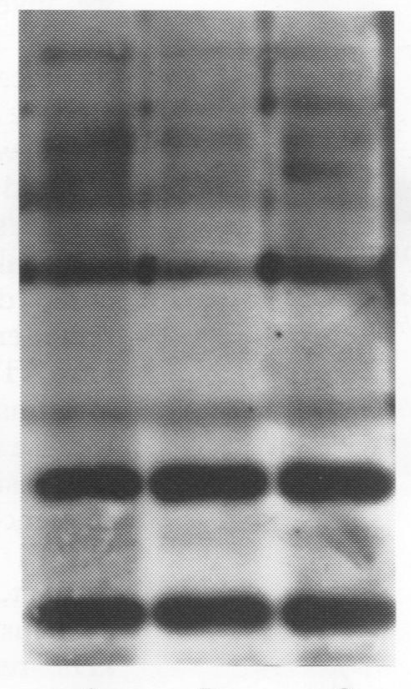

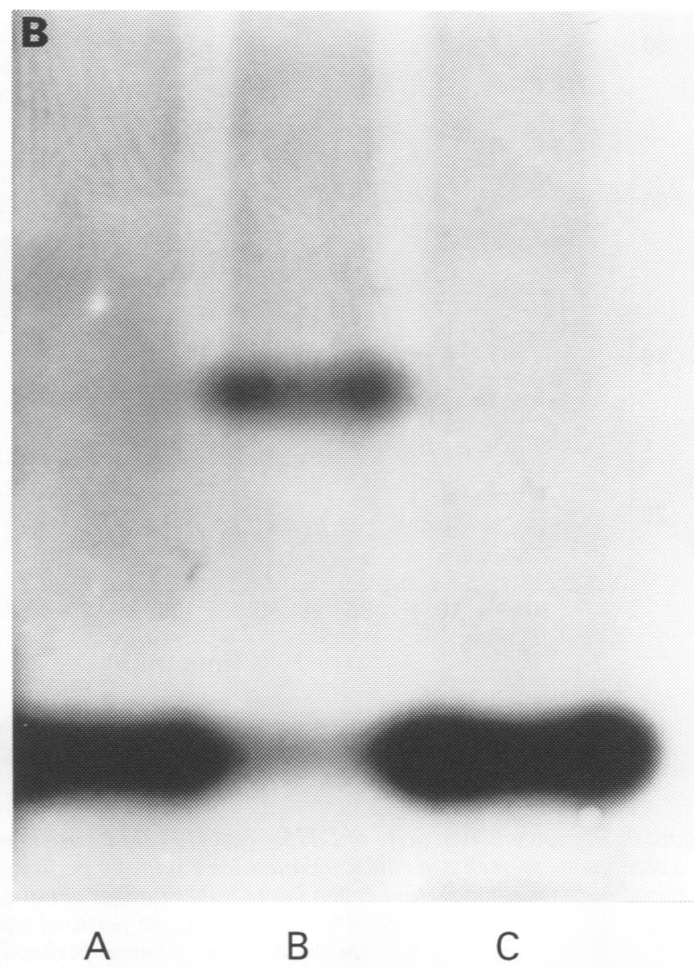

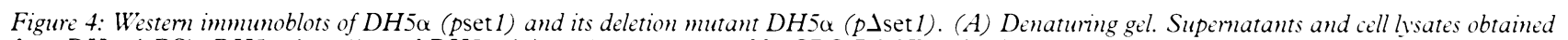

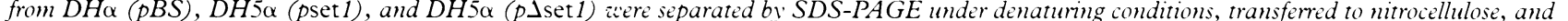
developed using rabbit antiserum to the active fraction obtained from Shigella flexneri $2 a$ strain. M4243azir. ${ }^{4}$ Lane A: DH5 $(p B S)$ cell lvsate; lane B:

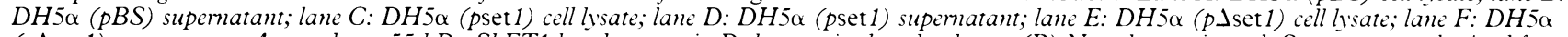
(p Jset 1) supernatant. Arrow show's $55 \mathrm{kDa}$ ShET1 band present in D, but not in the other lanes. (B) Non-denaturing gel. Supermatants obtained from

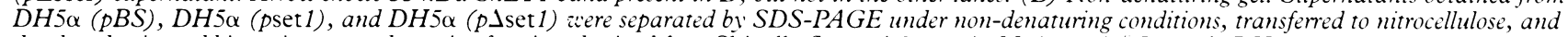
developed using rabbit antiserum to the active fraction obtained from Shigella flexneri $2 a$ strain M4243azir. Lane A: DH5 (pBS); lane B: DH5 $\alpha$ (pset 1); lane C: DH5a (p」set1). Note the presence of ShET1 band in B, but not A and C. (C) Western immunoblot developed zwith anti-ShET1 $A$

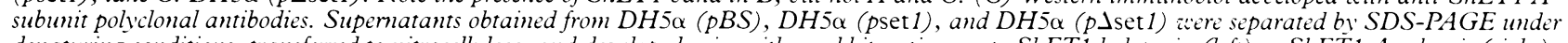
denaturing conditions, transferred to nitrocellulose, and developed using either rabbit antisemum to ShET1 holotoxin (left) or ShET1 A subunit (right).

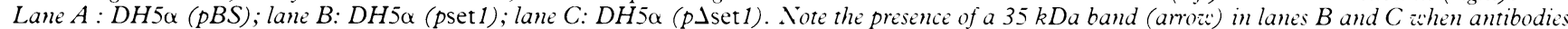
against the holotoxin w'ere used, whereas this band was not detected with antibodies against the ShET1 $A$ subunit.

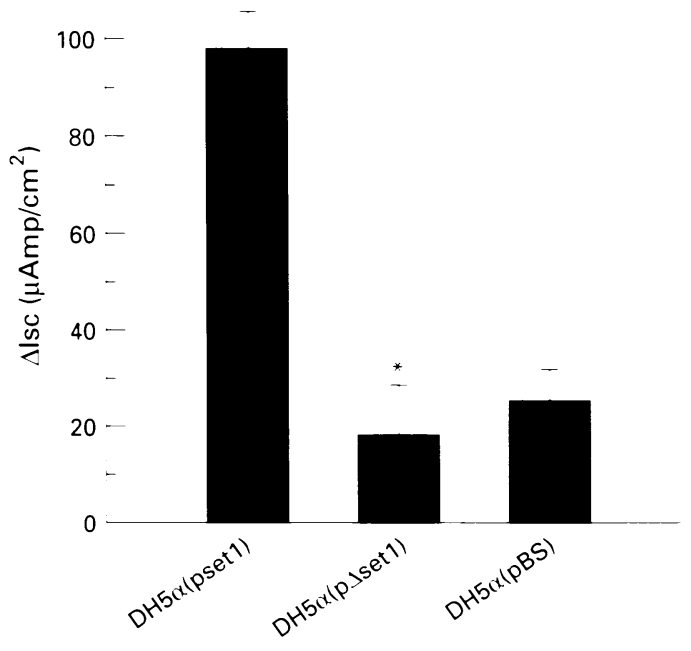

Figure 5: Enterotoxic effect of ShET1 deletion mutant in Ussing chambers. Peak increments in Isc after addition of supernatants obtained from DH5a (pset 1) (ShET1

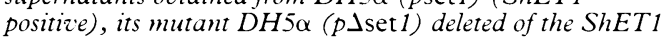

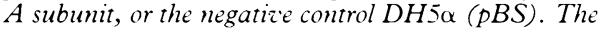
ShET1 deletion mutant failed to induce the rise in Isc found with ShET1 containing supermatants. ${ }^{*} p<0.01(n=5$ animals). concentrations of purified toxin obtained by electroelution were added to the mucosal side of rabbit ileum mounted in Ussing chambers and changes in Isc recorded. ShET 1 induced a dose dependent increment in Isc that reached its plateau at a toxin concentration of $4 \times 10^{\circ}$ $\mathrm{M}$ and an $\mathrm{ED}_{50}$ of $9 \times 10^{*} \mathrm{M}$ (Fig 7 ).

\section{Discussion}

Shigella flexneri $2 \mathrm{a}$ is one of the most common serotypes causing bacillary dysentery worldwide. ${ }^{22-25}$ The ability to invade and spread within the epithelial cells and thereby to cause dysentery has always been considered the cardinal mechanism of pathogenicity of Shigella flexneri. ${ }^{2-6}$ Nevertheless, the clinical presentation of shigellosis, with an initial phase of watery diarrhoea that in mild cases may represent the only gastrointestinal symptom, would predict the existence of enterotoxins. ${ }^{i}$ Further evidence of the involvement of other pathogenic factors (other than associated with shigella invasion and multiplication within the 


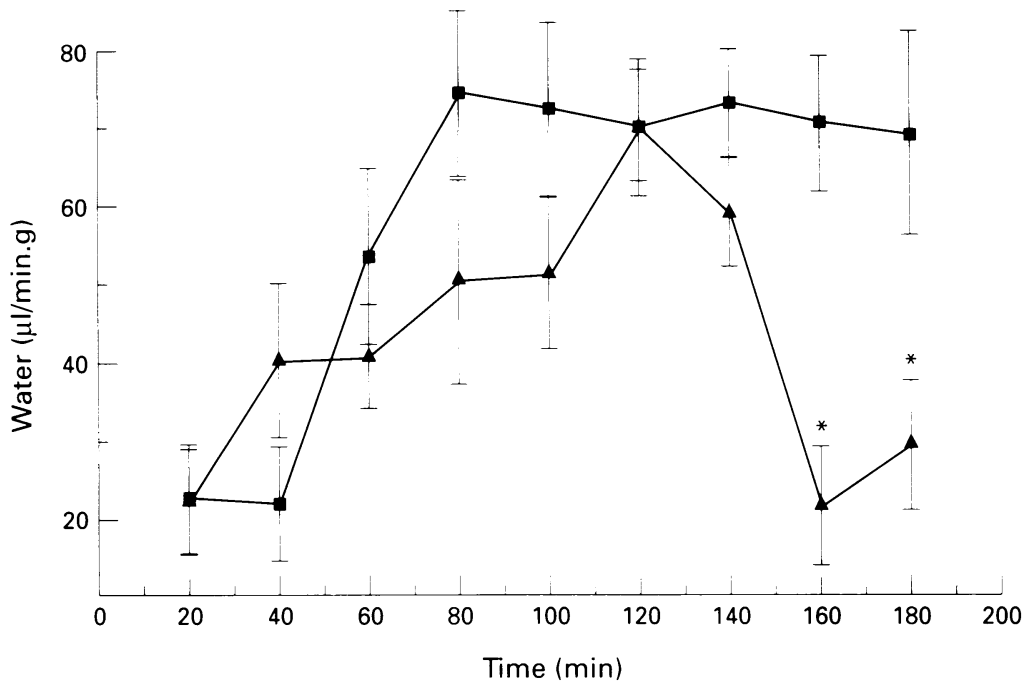

Perfusion solution

\section{Perfusion solution + supernatant}

Figure 6: In vivo enterotoxicity of $\mathrm{DH} 5 \alpha$ ( $p$ set 1) and its deletion mutant $\mathrm{DH} 5 \alpha$ ( $\mathrm{p} \Delta \mathrm{set} 1$ ). Two contiguous segments of rabbit jejunum were initially perfused with the saline solution

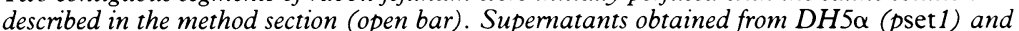
$D H 5 \alpha(p \Delta s e t 1)$ were then added to the solution and perfused separately (solid bar). After 60 minutes the addition of the supernatants, the segment perfused with ShET1 containing supernatant $(\mathbf{\Delta})$ showed a significant reduction in water absorption compared with the segment perfused with the toxin deleted of its $A$ subunit ( $\square) .{ }^{*} p<0.05$ ( $n=3$ animals).

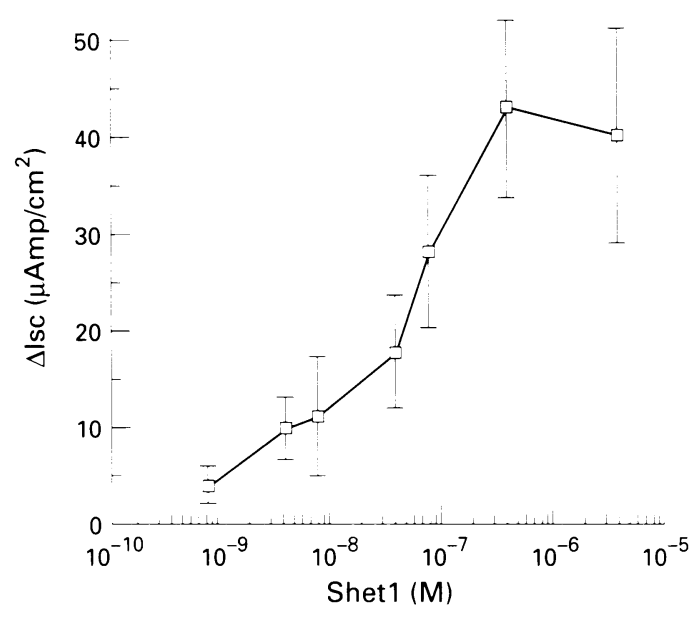

Figure 7: ShET1 dose response curve. Increasing concentrations of purified ShET1 obtained as described in Methods were added to the mucosal side of rabbit ileum mounted in Ussing chambers and peak increments in Isc recorded. No significant changes were found up to a toxin concentration of $10^{-8} \mathrm{M}$. Higher concentrations induced a dose dependent increment in Isc, with $50 \%$ of the effective dose $\left(E D_{5,}\right)$ at $9 \times 10^{-8} \mathrm{M}$ and a maximal dose of $4 \times 10^{-6}$ $M$. Further additions of the toxin did not induce significant changes in Isc ( $n=4$ animals).

enterocyte) come from recent vaccine trials. Lindberg and coworkers engineered a live, aromatic dependent $S$ flexneri vaccine candidate by deleting the aro $\mathrm{D}$ gene. ${ }^{26}$ Although such a strain was able to invade epithelial cells, its ability to grow intracellularly was greatly reduced. ${ }^{27}$ When this vaccine was tested in volunteers, some of them experienced a self limiting watery diarrhoea, with no blood or mucus in their faeces. ${ }^{28}$

Another vaccine candidate was engineered by transfer of the $140 \mathrm{mDa}$ invasiveness plasmid from $S$ flexneri 5 and the chromosomal genes encoding the group and type specific $\mathrm{O}$

antigen of $S$ flexneri 2 a to Escherichia coli $\mathrm{K}-12 .{ }^{29}$ This vaccine also induced adverse reactions in $31 \%$ of vaccinees; among the side effects, watery diarrhoea was reported.

We have recently reported the elaboration of two distinct enterotoxins by Shigella flexneri $2 a,{ }^{9}{ }^{10}$ that may be responsible for the watery phase of shigellosis. We have shown that, when grown in iron depleted medium, enteroinvasive Escherichia coli (EIEC) elaborate an enterotoxin (EIET) that causes fluid accumulation in isolated ileal loops and an electrical response in Ussing chambers. ${ }^{12}$ Based on the similarities known to exist between EIEC and shigella, ${ }^{30}$ we investigated the possibility that Shigella flexneri 2a elaborates a similar toxin. Our group has recently cloned and sequenced the EIET gene. ${ }^{30}$ A virtually identical gene $(99 \%$ homology) was found on the $140 \mathrm{mDa}$ invasiveness plasmid of Shigella flexneri $2 \mathrm{a}$ and gene probe studies showed that this gene is widely present in all shigella serotypes. ${ }^{10}$ Surprisingly, plasmid cured derivatives of Shigella flexneri 2a still retain enterotoxic activity, both in vivo and in vitro. ${ }^{9}$ The combination of genetic analysis and intestinal electrophysiology allowed us to localise the chromosomal genes responsible for this residual enterotoxicity. ${ }^{9}$ Analysis of the sequence of the cloned genes responsible for ShET1 enterotoxic activity disclosed two distinct, yet contiguous orfs, encoding for two proteins of predicted $\mathrm{MW}$ of $7 \mathrm{kDa}$ and 20 $\mathrm{kDa}$, respectively. The common $\mathrm{A}: \mathrm{B}_{\mathrm{n}}$ active binding subunit motif often found among bacterial enterotoxins, including cholera toxin (CT), ${ }^{18}$ heat labile enterotoxin (LT) of enterotoxigenic $E$ coli, ${ }^{19}$ and shiga toxin of $S$ dysenteriae 1,2021 may be reflected in these data. The apparent molecular sizes of active material as predicted by both the gene sequencing and the western blot experiments, ${ }^{9}$ are consistent with such stoichiometries based on the sizes of the $\mathrm{A}(20-32 \mathrm{kDa})$ and $\mathrm{B}(7-11 \mathrm{kDa})$ subunits of these recognised enterotoxins. By extension, a holotoxin consistent with a size of $55 \mathrm{kDa}$ and an $A_{1}: B_{5}$ structure would be predicted by these conventions. These tentative configurations also satisfy the usual requirements for both a binding and an active domain that allow the enterotoxin to attach and gain entrance to enterocytes and to initiate events that culminate in intestinal secretion. The data presented in this paper suggest that, as for other enterotoxins, the ShET1 A subunit is responsible for the secretory activity, whereas the $B$ subunits may be involved in the irreversible binding of the toxin to the enterocyte receptor. With regular discoveries of additional members of a family of CT-/LT-like toxins among salmonella, pseudomonas, campylobacter, aeromonas, and other genera, new combinations of A:B structures seem likely to emerge.

It is intriguing that none of the currently identified intracellular messengers of intestinal secretion is involved in ShET1 enterotoxicity. However, the possibility that intestinal secretagogues may act via new intracellular modulators is emerging both for bacterial enterotoxins (RDEC enterotoxin, F Raimondi and A Fasano, unpublished data) and inflam- 
matory mediators such as adenosine and its non-metabolised analogues phenylisopropyl adenosine (PIA) and N-ethlylcarboxamidoadenosine (NECA). ${ }^{31-33}$ This new finding may open unexplored avenues on the intracellular signalling involved in the intestinal fluid and electrolyte transepithelial regulation. Experiments to confirm this hypothesis are currently in progress in our laboratory.

This work was supported by National Institute of Health grants 1 RO1DK48373 and 1RO1AI35740 to AF and NO1AI45251, 1UO1AI35948-01, and RO1AI29471 to MML.

1 Shiga K. Ueber den Dysenterriebacillus (Bacillus dysenteriae). Zentbl Bakt ParasitKde Abt I Orig 1898; 24: 817-24.

2 Makino S, Sasakawa C, Yoshikawa M. Genetic relatedness of the basic replicon of the virulence plasmid in the shigellae and enteroinvasive Escherichia coli. Microb Pathog 1988; 5: 267-74.

3 Sansonetti PJ, Kopecko DJ, Formal SB. Involvement of a plasmid in the invasive ability of $\mathrm{S}$ flexneri. Infect Immun 1982; 35: 852-60.

4 Hale TL, Sansonetti PJ, Schad PA, Austin S, Formal SB. Characterization of virulence plasmids and plasmidassociated outer membrane proteins in S flexneri, Shigella sonnei, and Escherichia coli. Infect Immun 1983; 40: 340-50.

5 Pal T, Formal SB, Hale TL. Characterization of virulence marker antigen of Shigella spp and enteroinvasive Escherichia coli. $\mathcal{f}$ Clin Microbiol 1989; 27: 561-3

6 Venkatesan MM, Buysse JM, and Kopecko DJ. Characterization of invasion plasmid antigen genes (ipaBCD) from S flexneri. Proc Natl Acad Sci USA 1988; 85: 9317-21.

7 Taylor DN, Echeverria P, Pal T, Sethabutr O, Saiborisuth S, Srichamorn S, et al. The role of Shigella spp, enteroinvasive Escherichia coli and other enteropathogens as causes of childhood dysentery in Thailand. F Infect Dis causes of childhood

8 Taylor DN, Echeverria P, Sethabutr O, Pitarangsi C, Leksomboon U, Blacklow $\mathrm{N}$, et al. Clinical and microbiological features of Shigella and enteroinvasive Escherichia coli infections detected by DNA hybridization. $f$ Clin Microbiol 1988; 26: 1362-6.

9 Fasano A, Noriega FR, Maneval RD Jr, Chanasongcram S, Russell R, Guandalini S, Levine MM. Shigella enterotoxin 1: an enterotoxin of Shigella flexneri 2a active in rabbit small intestine. $\mathcal{f}$ Clin Invest 1995; 95: 2853-61.

10 Nataro JP, Seriwatana J, Fasano A, Maneval DR Jr, Guers LD, Noriega FR, et al. Identification and cloning of a novel plasmid-encoded enterotoxin in enteroinvasive $\mathrm{E}$ coli and Shigella. Infect Immun 1995; 63: 4721-8.

11 Noriega FR, Ming Liao F, Formal SB, Fasano A, Levine MM. Prevalence of Shigella enterotoxin 1 (ShET1) among Shigella clinical isolates of diverse serotypes. F Infect Dis 1995; 172: 1408-10.

12 Fasano A, Kay BA, Russell BG, Maneval DR Jr, Levine MM. Enterotoxin and cytotoxin production by enteroinvasive Escherichia coli. Infect Immun 1991; 58: 3717-23.

13 Bradford MM. A rapid and sensitive method for the quantitation of micrograms quantities of protein utilizing the principle of protein dye binding. Anal Biochem 1976; the principle $248-54$.

14 Ko YH, Thomas PJ, Delannoy MR, Pedersen PL. The cystic fibrosis transmembrane canductance regulator. Over expression, purification, and characterization of wild type and DF508 mutant forms of the first nucleotide binding fold in fusion with the maltose-binding protein. 7 Biol Chem 1993; 268: 24330-8.

15 Fasano A, Hokama Y, Russell BG, Morris JG Jr. Diarrhea in ciguatera fish poisoning: preliminary evaluation of pathophysiological mechanisms. Gastroenterology 1991; 100: 471-6.

16 Field M, Fromm D, McColl I. Ion transport in rabbit ileal mucosaI. $\mathrm{Na}$ and $\mathrm{Cl}$ fluxes and short circuit current. $\mathrm{Am}$ f Physiol 1971; 220: 1388-96.

17 Sladen GE, Harries JT. Studies on the effects of unconjugated dihydroxy bile salts on rat small intestinal function in vivo. Biochem Biophys Acta 1972; 288: 443-56.

18 LoSpalluto JJ, Finkelstein RA. Chemical and physical properties of cholera exo-enterotoxin (choleragen) and its spontaneously formed toxoid (choleragenoid). Biochem Biophys Acta 1972; 257: 158-66.

19 Clements JD, Flint DC, Klipstein AF. Immunological and physicochemical characterization of heat-labile enterotoxins isolated from two strains of Escherichia coli. Infect Immun 1982; 38: 806-809.

20 Olsnes S, Reisbig R, Eiklid K. Subunit structure of Shigella cytotoxin. F Biol Chem 1981; 256: 8732-8.

21 Seidah NG, Donohue-Rolfe A, Lazure C, Auclair F, Keusch GT, Chretein M. Complete amino acid sequence of Shigella toxin-B chain. A novel polypeptide containing 69 amino acids and one disulfide bridge. 7 Biol Chem 1986; 261: 13928-31.

22 Casalino M, Yusuf MW, Nicoletti $M$, et al. A two year study of enteric infections associated with diarrhoeal diseases in children in Urban Somalia. Trans $R$ Soc Trop Med Hyg 1988; 82: 637-41.

23 Ferrecio C, Prado V, Ojeda A, Cayyazo M, Abrego P, Guers L, Levine MM. Epidiemiologic patterns of acute diarrhea and endemic Shigella infections in children in a poor periurban setting in Santiago, Chile. Am $\mathcal{F}$ Epidemiol 1991; 134: 614-27.

24 Mata LJ. The children of Santa Maria Cauque: a prospective field study of health and growth. Cambridge, MA: MIT Press, 1978.

25 Piechaud D, Szturm-Rubistein S, D'Hauteville H, et al. Epidemiologie de la dysenterie bacillaire a Dijibouti. Bulletin de la Societe de Pathologie Exotique et de ses Filiales 1971; 64: 37-42.

26 Lindberg AA, Karnell A, Pal T, Sweiha H, Hultenby K, Stocker BA. Shigella flexneri strain for use as a live Stocker BA. Shigella flexneri strain for
vaccine. Microb Pathog 1990; 8: 433-40.

27 Karnell A, Stocker BAD, Katadura S, Reinholt FP, Lindberg AA. Live oral auxotrophic Shigella flexneri SFL 12 vaccine with a deleted aroD gene; characterization and monkey protection studies. Vaccine 1992; 10: 389-94.

28 Karnell A, Li A, Zhao CR, Karlsson K, Nguyen BM, Lindberg AA. Safety and immunogenicity study of the auxotrophic Shigella flexneri 2a vaccine SFL 1070 with a deleted aro $\mathrm{D}$ gene in adult Swedish volunteers. Vaccine 1995; 1: 88-99.

29 Kotloff KL, Herrington DA, Hale TL, et al. Safety, immunogenicity and efficacy in monkeys and humans of invasive Escherichia coli K-12 hybrid vaccine candidates expressing Shigella flexneri 2a somatic antigen. Infect expressing Shigella flexner

30 Levine MM. Escherichia coli that cause diarrhea: enterotoxigenic, enteropathogenic, enteroinvasive, enterohemorrhagic, and enteroadherent. F Infect Dis 1987; 155: 377-89.

31 Barret KE, Dharmsathaphorn K. Mechanisms of chloride secretion in a colonic epithelial cell line. In: Lebenthal E, Duffey M, eds. Texbook of secretory diarrhea. New York: Raven Press, 1990: 59-66.

32 Barret KE, Cohn JA, Huott PA, Wasserman SI, Dharmsathaphorn $\mathrm{K}$. Immune-related intestinal $\mathrm{Cl}^{-}$ secretion. II. Effect of adenosine on the $\mathrm{T}_{84}$ cell line. Am f Physiol 1990; 258: C902-12.

33 Barrett KE, Shin JA, MacMartin KA, Dharmsathaphorn K. A possible role for arachidonic acid in the chloride secretory response induced by adenosine in $T_{84}$ cells [abstract]. Gastroenterology 1988; 94: A24. 